\title{
Interactive Cloth Rendering of Microcylinder Appearance Model under Environment Lighting
}

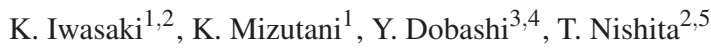 \\ ${ }^{1}$ Wakayama University, ${ }^{2}$ UEI Research, ${ }^{3}$ Hokkaido University, ${ }^{4}$ JST CREST, ${ }^{5}$ Hiroshima Shudo University
}

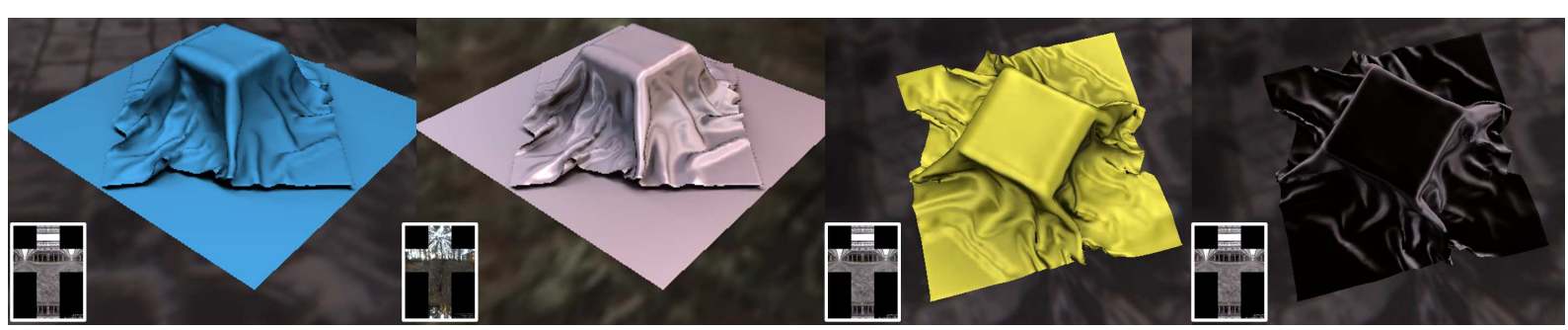

Figure 1: Our method can render various cloth fabrics with microcylinder appearance model under environment lighting at interactive frame rates. The insets show the environment maps. From left to right, cloth fabrics are linen plain, polyester satin charmuse, silk crepe de chine, velvet.

\begin{abstract}
This paper proposes an interactive rendering method of cloth fabrics under environment lighting. The outgoing radiance from cloth fabrics in the microcylinder model is calculated by integrating the product of the distant environment lighting, the visibility function, the weighting function that includes shadowing/masking effects of threads, and the light scattering function of threads. The radiance calculation at each shading point of the cloth fabrics is simplified to a linear combination of triple product integrals of two circular Gaussians and the visibility function, multiplied by precomputed spherical Gaussian convolutions of the weighting function. We propose an efficient calculation method of the triple product of two circular Gaussians and the visibility function by using the gradient of signed distance function to the visibility boundary where the binary visibility changes in the angular domain of the hemisphere. Our GPU implementation enables interactive rendering of static cloth fabrics with dynamic viewpoints and lighting. In addition, interactive editing of parameters for the scattering function (e.g. thread's albedo) that controls the visual appearances of cloth fabrics can be achieved.
\end{abstract}

Categories and Subject Descriptors (according to ACM CCS): I.3.7 [Computer Graphics]: Three-Dimensional Graphics and Realism-Color, shading, shadowing, and texture

\section{Introduction}

Photorealistic rendering of cloth fabrics is one of the most important research topics in the field of Computer Graphics, and is used for many applications such as cloth design, interior design, games, and web shopping applications. For such applications, realistic rendering of cloth fabrics under realworld, complex lighting at interactive frame rates and edit- ing parameters for scattering effects on threads to change the appearance of cloth fabrics are desired.

This paper proposes an interactive rendering method of cloth fabrics made from interwoven threads illuminated from dynamic environment lighting. Our method employs the microcylinder appearance model [Sad11, SBdDJ13] as the shading model of cloth fabrics since the microcylinder model has several nice properties. The microcylinder model 
can represent various appearances of cloth fabrics, from diffuse matte reflections to highly anisotropic glossy reflections, which are validated by comparing the measured data. The microcylinder model is robust, practical, and easy to control by changing a small number of intuitive parameters for scattering functions.

In the microcylinder model, the outgoing radiance from cloth fabrics is calculated by integrating the product of the lighting, the visibility function, the scattering function, and the weighting function calculated from the geometric structure of interwoven threads. The main difficulty of interactive cloth rendering resides in the integral of high-frequency complex functions, limiting the previous rendering methods [RZL $\left.{ }^{*} 10, \mathrm{XMR}^{*} 11\right]$ to ignoring the visibility function or limiting to a simple lighting model [SBdDJ13].

To alleviate this difficulty, our method proposes an efficient calculation method of the quadruple product integral for cloth rendering of static scenes. The environment lighting is represented with a linear combination of spherical Gaussians (SGs). The quadruple product integral of the SG, the visibility function, the weighting function and the scattering function is approximated by the product of SG convolved weighting function and the triple product integral of the SG, the visibility function and the scattering function. The SG convolved weighting functions are precomputed for various geometric structures of threads and SGs, and easily calculated from the precomputed table in the run-time. To compute the triple product, our method decomposes the SG into two circular Gaussians. The scattering function in the microcylinder model can be represented by the product of a circular Gaussian and smooth functions. By factoring out the smooth functions, the triple product integral is simplified to the product integral of two circular Gaussians and the visibility function, which can be easily computed by using signed distance function (SDF) from the visibility boundary where the visibility changes, and the gradient of SDF.

\section{Background}

\subsection{Previous Methods}

Shading model for cloth: To render cloth fabrics efficiently, many cloth shading models have been proposed. Yasuda et al. proposed a simple shading model for cloth to reproduce the glossy reflection of cloth [YYTI92]. Westin et al. developed an estimation method of BRDFs from microscale cloth geometries [WAT92]. Daubert et al. precomputed the reflected radiance from implicit geometric models of knit or weaving patterns and modeled spatially varying BRDFs of fabrics using Lafortune BRDF models [DLHpS01]. Microfacet BRDF models proposed by Ashikhmin et al. [APS00] are used to shade cloth fabrics such as velvet and satin. Realtime rendering methods [AMTF03, Kan10] and synthesizing method from single-view captured images [WZT*08] have been proposed for microfacet BRDF models.
In recent years, Irawan et al. proposed a novel and realistic reflectance model for woven fabrics [IM12]. This method, however, does not take into account shadowing and masking effects. Sadeghi et al. introduced a versatile reflectance model for cloth fabrics [SBdDJ13]. All these methods mainly focus on developing a comprehensive reflectance model for cloth fabrics and do not propose an efficient rendering method of cloth fabrics under environment lighting.

BTF representation for cloth: Several methods have been proposed to render realistic cloth fabrics under environment lighting by using bidirectional texture functions (BTFs). Sattler et al. [SSK03] proposed an interactive rendering method of cloth fabrics under environment lighting using BTFs. This method, however, requires a huge amount of precomputed data per BTF. To address this problem, Muller et al. [MMK04] proposed a Local-PCA encoded BTF method. Since this method employs Precomputed Radiance Transfer with spherical harmonics [SKSO2], the environment lighting is limited to low-frequency lighting.

Volumetric approaches: $\mathrm{Xu}$ et al. proposed a realistic rendering method for knitwear using the lumislice $\left[\mathrm{XCL}^{*} 01\right]$. This method, however, does not achieve interactive rendering for environment lighting. In recent years, volumetric approaches to render realistic fabrics have been proposed [JAM*10, SKZ11, ZJMB11, ZJMB12]. Although these methods can render realistic fabrics with high resolution volume data by using path tracing methods, these methods do not achieve interactive rendering. While Zhao et al. accelerated the path tracing of structured volumetric models by using the modular flux transfer method [ZHRB13], interactive rendering of fabrics cannot be achieved. Hašan and Ramamoorthi proposed an interactive editing method of volumetric materials (e.g. scarf) [HR13]. This method, however, is limited to fixed lighting, while our method can render cloth fabrics under dynamic environment lighting.

Hair rendering under environment lighting: We briefly review the rendering methods of hair under environment lighting which are similar to cloth rendering. Ren et al. proposed an interactive hair rendering method under environment lighting [RZL $\left.{ }^{*} 10\right]$. Xu et al. extended Ren's method to achieve interactive editing of scattering parameters for hair [XMR*11]. Although these methods can render realistic hair taking into account scattering effects at hair fibers under all-frequency environment lighting, these methods do not take into account shadows from occluders. Therefore, it is difficult to apply these methods to cloth rendering in which shadows from the cloth itself are necessary to enhance the realism of the cloth.

\subsection{Definition of Gaussian functions and terminology}

To render cloth fabrics under environment lighting efficiently, our method uses spherical Gaussian (SG) $G$, circular 


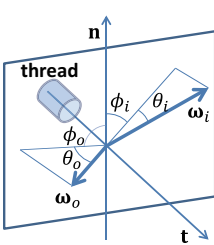

(a)

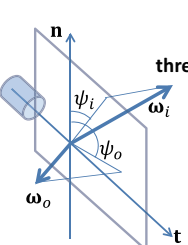

(b)

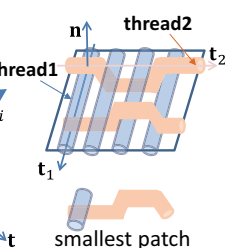

(c)

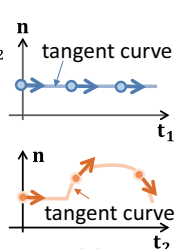

(d)
Figure 2: Notation of angles for microcylinder model. (a) Longitudinal angles $\theta_{i}, \theta_{o}$ are angles from the plane perpendicular to the thread tangent $\mathbf{t}$. Azimuthal angles $\phi_{i}, \phi_{o}$ are angles between $\mathbf{n}$ and projected $\boldsymbol{\omega}_{i}, \boldsymbol{\omega}_{o}$ onto the plane perpendicular to $\mathbf{t}$. $(b) \psi_{i}, \psi_{o}$ are angles between $\mathbf{n}$ and projected $\boldsymbol{\omega}_{i}, \boldsymbol{\omega}_{o}$ onto the plane spanned by $\mathbf{n}$ and $\mathbf{t}$. (c) weaving pattern (top) and smallest patch (bottom). (d) tangent curves of two threads in the smallest patch.

Gaussian $g^{c}$, Gaussian $g$, and normalized Gaussian $g^{u}$ as:

$$
\begin{aligned}
G(\boldsymbol{\omega} ; \boldsymbol{\xi}, \boldsymbol{\sigma}) & =\exp \left(\frac{2}{\sigma^{2}}(\boldsymbol{\omega} \cdot \boldsymbol{\xi}-1)\right), \\
g^{c}(x ; \mu, \sigma) & =\exp \left(\frac{2}{\sigma^{2}}(\cos (x-\mu)-1)\right), \\
g(x ; \mu, \sigma) & =\exp \left(-\frac{(x-\mu)^{2}}{\sigma^{2}}\right), \\
g^{u}(x ; \mu, \sigma) & =g(x ; \mu, \sigma) /(\sqrt{\pi} \sigma),
\end{aligned}
$$

where $\boldsymbol{\omega}$ is a unit direction vector, $\boldsymbol{\xi}, \sigma$, and $\mu$ represent the lobe axis (unit vector), the bandwidth, and the mean, respectively.

The outgoing radiance of cloth is calculated in the local coordinate system of tangent $\mathbf{t}$ and normal $\mathbf{n}$ as shown in Fig. 2. Longitudinal angle $\theta$ is the angle from the plane perpendicular to $\mathbf{t}$, and $\phi$ is the azimuthal angle from $\mathbf{n}$. Longitudinal angles and azimuthal angles for incident direction $\boldsymbol{\omega}_{i}$ and outgoing direction $\boldsymbol{\omega}_{o}$ are denoted as $\theta_{i}, \phi_{i}$ and $\theta_{o}, \phi_{o}$, respectively. Our method defines $\theta_{d}=\left(\theta_{i}-\theta_{o}\right) / 2$, $\theta_{h}=\left(\theta_{i}+\theta_{o}\right) / 2$, and $\phi_{d}=\phi_{i}-\phi_{o}$. As shown in Fig. 2, angles $\psi_{i}$ and $\psi_{o}$ are angles between $\mathbf{n}$ and the projection of $\boldsymbol{\omega}_{i}$ and $\boldsymbol{\omega}_{o}$ onto the plane spanned by $\mathbf{t}$ and $\mathbf{n}$, and $\psi_{d}$ is defined as $\psi_{i}-\psi_{o}$.

\subsection{Microcylinder Model}

In the microcylinder model [SBdDJ13], the threads of the cloth fabrics are modeled as microcylinders, and the cloth fabrics are modeled by interwoven microcylinders in two directions orthogonal to each other as shown in Fig. 2(c). The smallest portion of the weaving pattern is referred to as smallest patch (Fig. 2(c)). The cloth fabrics are modeled by repeating the smallest patch. The outgoing radiance $L\left(\boldsymbol{\omega}_{o}\right)$ from the cloth is calculated by $L\left(\boldsymbol{\omega}_{o}\right)=a_{1} L_{1}\left(\boldsymbol{\omega}_{o}\right)+$ $a_{2} L_{2}\left(\boldsymbol{\omega}_{o}\right)$, where $L_{1}$ and $L_{2}$ are outgoing radiances from two threads of the smallest patch, $a_{1}$ and $a_{2}$ are the area coverage ratios of two threads, respectively. For each thread $j(=1,2)$,

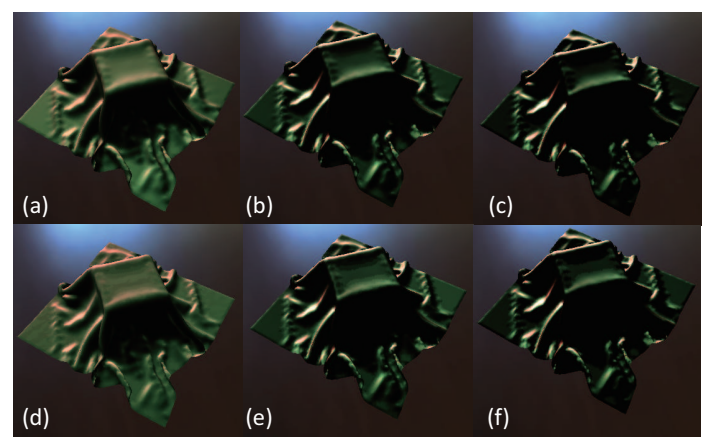

Figure 3: Comparison of our $S G$ convolution approximation of $W$ and the reference (unshadowed rendering) for silk-shot fabric that consists of red and green threads. The incident lighting is represented by a single $S G G\left(\boldsymbol{\omega}_{i},(0,1,0)^{T}, \sigma\right)$. The upper row images are the reference images and the bottom row images are rendered by using SVD compressed $T_{\mathbf{t}, \sigma}\left(\xi_{k}, \boldsymbol{\omega}_{o}\right) .(a)(d) 2 / \sigma^{2}=10,(b)(e) 2 / \sigma^{2}=100$, and $(c)(f)$ $2 / \sigma^{2}=1000$.

tangent $\mathbf{t}$ is defined by the tangent curve (Fig. 2(d)). Let $C_{j}$ be the set of tangent vectors obtained by sampling the tangent curve. The outgoing radiance $L_{j}\left(\boldsymbol{\omega}_{o}\right)$ of $j$-th thread is calculated by the following equation [Sad11]:

$L_{j}\left(\boldsymbol{\omega}_{o}\right)=\int_{\Omega} L\left(\boldsymbol{\omega}_{i}\right) \sum_{\mathbf{t} \in C_{j}} f_{s}\left(\mathbf{t}, \boldsymbol{\omega}_{i}, \boldsymbol{\omega}_{o}\right) W\left(\mathbf{t}, \boldsymbol{\omega}_{i}, \boldsymbol{\omega}_{o}\right) \cos \theta_{i} d \boldsymbol{\omega}_{i},(5)$

where $\Omega$ is a set of directions on the hemisphere, $L$ is the incident radiance, $f_{s}$ is the scattering function of thread and calculated by the sum of surface scattering function $f_{r, s}$ and volume scattering function $f_{r, v}$ as follows:

$$
\begin{aligned}
f_{s}\left(\mathbf{t}, \boldsymbol{\omega}_{i}, \boldsymbol{\omega}_{o}\right) & =\left(f_{r, s}\left(\mathbf{t}, \boldsymbol{\omega}_{i}, \boldsymbol{\omega}_{o}\right)+f_{r, v}\left(\mathbf{t}, \boldsymbol{\omega}_{i}, \boldsymbol{\omega}_{o}\right)\right) / \cos ^{2} \theta_{d}, \\
f_{r, s}\left(\mathbf{t}, \boldsymbol{\omega}_{i}, \boldsymbol{\omega}_{o}\right) & =F_{r}\left(\eta, \theta_{d}, \phi_{d}\right) \cos \left(\phi_{d} / 2\right) g^{u}\left(\theta_{h} ; 0, \gamma_{s}\right), \\
f_{r, v}\left(\mathbf{t}, \boldsymbol{\omega}_{i}, \boldsymbol{\omega}_{o}\right) & =F \frac{\left(1-k_{d}\right) g^{u}\left(\theta_{h} ; 0, \gamma_{v}\right)+k_{d}}{\cos \theta_{i}+\cos \theta_{o}} A,
\end{aligned}
$$

where $F_{r}$ is the Fresnel reflectance, $\eta$ is the refraction index of the thread, $F$ is the product of Fresnel transmittances, $k_{d}$ and $A$ are parameters for the isotropic term and the albedo.

Weighting function $W$ is calculated by the shadowing and masking term $M\left(\mathbf{t}, \boldsymbol{\omega}_{i}, \boldsymbol{\omega}_{o}\right)$ and the projection term $P\left(\mathbf{t}, \boldsymbol{\omega}_{i}, \boldsymbol{\omega}_{o}\right)$ as:

$$
W\left(\mathbf{t}, \boldsymbol{\omega}_{i}, \boldsymbol{\omega}_{o}\right)=M\left(\mathbf{t}, \boldsymbol{\omega}_{i}, \boldsymbol{\omega}_{o}\right) \cdot \frac{P\left(\mathbf{t}, \boldsymbol{\omega}_{i}, \boldsymbol{\omega}_{o}\right)}{\sum_{\mathbf{t}^{\prime} \in C_{1} \cup C_{2}} P\left(\mathbf{t}^{\prime}, \boldsymbol{\omega}_{i}, \boldsymbol{\omega}_{o}\right)} .
$$

By defining $D$ as $D(x, y, z)=(1-x) \cdot y \cdot z+x \cdot \min (y, z)$ to simplify the notation, the shadowing and masking term $M$ and the projection term $P$ are calculated by:

$M\left(\mathbf{t}, \boldsymbol{\omega}_{i}, \boldsymbol{\omega}_{o}\right)=D\left(g\left(\phi_{d} ; 0, \sigma\right), \max \left(\cos \phi_{i}, 0\right), \max \left(\cos \phi_{o}, 0\right)\right)$, $P\left(\mathbf{t}, \boldsymbol{\omega}_{i}, \boldsymbol{\omega}_{o}\right)=D\left(g\left(\psi_{d} ; 0, \sigma\right), \max \left(\cos \psi_{i}, 0\right), \max \left(\cos \psi_{o}, 0\right)\right)$, where $\sigma$ is a bandwidth parameter between $15^{\circ}$ and $20^{\circ}$. 


\section{Our Method}

Our method represents the environment lighting $L_{\text {env }}$ with a linear combination of SGs as $L_{\text {env }}\left(\boldsymbol{\omega}_{i}\right) \approx \sum_{k=1}^{K} L_{k} G\left(\boldsymbol{\omega}_{i} ; \boldsymbol{\xi}_{k}, \sigma_{k}\right)$ where $K$ is the number of SGs, $\xi_{k}, \sigma_{k}, L_{k}$ are $k$-th lobe axis, bandwidth, and coefficient, respectively. To simplify the explanation, $G\left(\boldsymbol{\omega}_{i} ; \boldsymbol{\xi}_{k}, \sigma_{k}\right)$ is represented as $G_{k}\left(\boldsymbol{\omega}_{i}\right)$. The incident radiance $L\left(\boldsymbol{\omega}_{i}\right)$ at the shading point of the cloth illuminated by environment lighting is calculated by $L_{e n v}\left(\boldsymbol{\omega}_{i}\right) V\left(\boldsymbol{\omega}_{i}\right)$ where $V\left(\boldsymbol{\omega}_{i}\right)$ is the visibility function that takes into account occlusions due to the cloth itself or other objects. By substituting this into Eq. (5), $L_{j}\left(\boldsymbol{\omega}_{o}\right)$ is calculated by the following equation:

$$
L_{j}\left(\boldsymbol{\omega}_{o}\right)=\sum_{k=1}^{K} L_{k} \sum_{\mathbf{t} \in C_{j}} B_{j, k}\left(\mathbf{t}, \boldsymbol{\omega}_{o}\right),
$$

where $B_{j, k}\left(\mathbf{t}, \boldsymbol{\omega}_{o}\right)$ is calculated by the following equation.

$B_{j, k}\left(\mathbf{t}, \boldsymbol{\omega}_{o}\right)=\int_{\Omega} G_{k}\left(\boldsymbol{\omega}_{i}\right) V\left(\boldsymbol{\omega}_{i}\right) f_{s}\left(\mathbf{t}, \boldsymbol{\omega}_{i}, \boldsymbol{\omega}_{o}\right) W\left(\mathbf{t}, \boldsymbol{\omega}_{i}, \boldsymbol{\omega}_{o}\right) \cos \theta_{i} d \boldsymbol{\omega}_{i}$.

\subsection{Convolution of spherical Gaussian and weighting function}

In the following, we describe the calculation method of $B_{j, k}\left(\mathbf{t}, \boldsymbol{\omega}_{o}\right)$. As described before, scattering function $f_{s}$ can be represented by $\theta_{i}, \phi_{i}, \theta_{o}, \phi_{o}$, and the product integral of $f_{s}$ and spherical Gaussian $G$ can be efficiently calculated by using the previous method [XMR*11]. However, this method does not consider the visibility function $V$ and therefore cannot be directly applied to the cloth rendering. Moreover, $W$ is a complex, high dimensional function and it is quite difficult to represent $W$ with spherical harmonics or Gaussian functions (please refer to Sec. 1 of the supplemental document).

To address this problem, our method factors out $W$ as:

$$
\begin{aligned}
B_{j, k}\left(\mathbf{t}, \boldsymbol{\omega}_{o}\right) & \approx \frac{\int_{\Omega} G_{k}\left(\boldsymbol{\omega}_{i}\right) W\left(\mathbf{t}, \boldsymbol{\omega}_{i}, \boldsymbol{\omega}_{o}\right) d \boldsymbol{\omega}_{i}}{\int_{\Omega} G_{k}\left(\boldsymbol{\omega}_{i}\right) d \boldsymbol{\omega}_{i}} \\
& \times \int_{\Omega} G_{k}\left(\boldsymbol{\omega}_{i}\right) V\left(\boldsymbol{\omega}_{i}\right) f_{s}\left(\mathbf{t}, \boldsymbol{\omega}_{i}, \boldsymbol{\omega}_{o}\right) \cos \theta_{i} d \boldsymbol{\omega}_{i} .(9)
\end{aligned}
$$

Then our method defines the factored out component $T\left(\mathbf{t}, \boldsymbol{\xi}_{k}, \sigma_{k}, \boldsymbol{\omega}_{o}\right)$ as the following equation:

$$
T\left(\mathbf{t}, \boldsymbol{\xi}_{k}, \sigma_{k}, \boldsymbol{\omega}_{o}\right)=\frac{\int_{\Omega} G_{k}\left(\boldsymbol{\omega}_{i}\right) W\left(\mathbf{t}, \boldsymbol{\omega}_{i}, \boldsymbol{\omega}_{o}\right) d \boldsymbol{\omega}_{i}}{\int_{\Omega} G_{k}\left(\boldsymbol{\omega}_{i}\right) d \boldsymbol{\omega}_{i}} .
$$

Since $W$ depends on the geometric structure of the smallest patch, our method precomputes $T$ for various SGs and fetches it in the rendering. However, $T$ is a high dimensional function and it would require a huge amount of the precomputed data. Our method precomputes $T_{\mathbf{t}, \sigma}\left(\boldsymbol{\xi}_{k}, \boldsymbol{\omega}_{o}\right)$ for each sample tangent vector and discretized bandwidths, and compresses $T_{\mathbf{t}, \sigma}\left(\boldsymbol{\xi}_{k}, \boldsymbol{\omega}_{o}\right)$ using the Singular Value Decomposition. In our experiments, $T_{\mathbf{t}, \sigma}\left(\boldsymbol{\xi}_{k}, \boldsymbol{\omega}_{o}\right)$ can be accurately approximated with a small number of singular values. Fig. 3

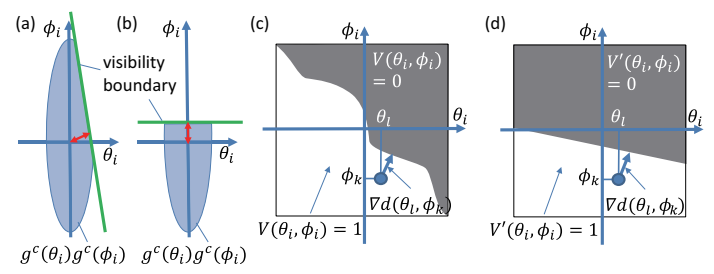

Figure 4: $(a)(b)$ : Product of two circular Gaussians and visibility function (blue ellipsoid cut by green line) differs even if the signed distance (red arrows) is same. $(c)(d)$ : Visibility function near $\left(\theta_{l}, \phi_{k}\right)$ is approximated by linear visibility function $V^{\prime}$ defined by signed distance and the gradient of SDF.

shows the validity of our approximation. Top images in Fig. 3 show the ground truth and bottom images are rendered by using SVD compressed $T_{\mathbf{t}, \sigma}\left(\boldsymbol{\xi}_{k}, \boldsymbol{\omega}_{o}\right)$. As shown in Fig. 3, our approximation method provides visually convincing results and the differences are subtle.

Next, we describe the calculation method for the latter integral in Eq. (9). To simplify the explanation, we describe the calculation method for the surface scattering component $f_{r, s}$, while that for the volume scattering component $f_{r, v}$ can be computed in the similar way. Let us define the latter integral in Eq. (9) as $I_{S}$. By substituting Eq. (6) into $I_{S}, I_{S}$ is calculated by the following equation:

$I_{s}=\int_{\Omega} G_{k}\left(\boldsymbol{\omega}_{i}\right) V\left(\boldsymbol{\omega}_{i}\right) F_{r}\left(\eta, \theta_{d}, \phi_{d}\right) \cos \left(\frac{\phi_{d}}{2}\right) g^{u}\left(\theta_{h} ; 0, \gamma_{s}\right) \frac{\cos \theta_{i}}{\cos ^{2} \theta_{d}} d \boldsymbol{\omega}_{i}$.

To compute $I_{s}, G_{k}\left(\boldsymbol{\omega}_{i}\right)$ is decomposed into the product of two circular Gaussians:

$$
G_{k}\left(\boldsymbol{\omega}_{i}\right)=g^{c}\left(\theta_{i}, \theta_{k}, \sigma_{k}\right) g^{c}\left(\phi_{i} ; \phi_{k}, \sigma_{k}^{\prime}\right),
$$

where $\theta_{k}, \phi_{k}$ are angles of $\boldsymbol{\xi}_{k}$ and $\sigma_{k}^{\prime}=\sigma_{k} / \sqrt{\cos \theta_{i} \cos \theta_{k}}$, respectively. Normalized Gaussian $g^{u}\left(\theta_{h}, 0, \gamma_{s}\right)$ is accurately approximated by circular Gaussian $g^{c}\left(\theta_{i} ;-\theta_{o}, 2 \gamma_{s}\right) / \sqrt{\pi} \gamma_{s}$ (the derivation is described in Sec. 2 of the supplemental document). Since the product of two circular Gaussians is closed in the circular Gaussian, the product of $g^{c}\left(\theta_{i} ; \theta_{k}, \sigma_{k}\right)$ and $g^{c}\left(\theta_{i} ;-\theta_{o}, 2 \gamma_{s}\right) /\left(\sqrt{\pi} \gamma_{s}\right)$ is replaced with another circular Gaussian $g^{c}\left(\theta_{i}, \theta_{l}, \sigma_{l}\right)$ multiplied by scaling coefficient $\alpha$ as:

$$
g^{c}\left(\theta_{i} ; \theta_{k}, \sigma_{k}\right) g^{c}\left(\theta_{i} ;-\theta_{o}, 2 \gamma_{s}\right) /\left(\sqrt{\pi} \gamma_{s}\right)=\alpha \cdot g^{c}\left(\theta_{i}, \theta_{l}, \sigma_{l}\right) \text {. }
$$

To simplify the notation, let us define $C\left(\theta_{i}, \phi_{i}\right)$ as $\alpha F_{r}\left(\eta, \theta_{d}, \phi_{d}\right) \cos \left(\phi_{d} / 2\right) \cos ^{2} \theta_{i} / \cos ^{2} \theta_{d}$. Since Fresnel term $F_{r}\left(\eta, \theta_{d}, \phi_{d}\right)$ and cosine functions $\cos \left(\phi_{d} / 2\right)$ and $\cos \theta_{i} / \cos ^{2} \theta_{d}$ are smooth compared to circular Gaussians, $C$ can be factored out from the integral as follows:

$I_{s} \approx C\left(\theta_{l}, \phi_{k}\right) \iint_{-\frac{\pi}{2}}^{\frac{\pi}{2}} g^{c}\left(\theta_{i} ; \theta_{l}, \sigma_{l}\right) g^{c}\left(\phi_{i} ; \phi_{k}, \sigma_{k}^{\prime}\right) V\left(\theta_{i}, \phi_{i}\right) d \theta_{i} d \phi_{i}$.

In the following, we describe the calculation method of triple product integral of two circular Gaussians and the visibility function. 


\subsection{Integrating circular Gaussians with SDF gradient}

To compute the product integral with the visibility function efficiently, spherical signed distance functions (SSDF) have been used for SGs [XJF*08, $\mathrm{WRG}^{*} 09$, IFDN12,IDN12, WPC $\left.^{*} 13\right]$. Although SSDF works well for SGs, SSDF cannot be directly applied to the product integral of two circular Gaussians and the visibility function. The product of two circular Gaussians can be an anisotropic function since the bandwidths of two circular Gaussians can differ. As shown in Eq. (1), SG is an isotropic function with respect to the lobe axis, only the spherical singed distance to the lobe axis matters. For anisotropic functions, however, not only the spherical signed distance but also the direction to the nearest visibility boundary are required to accurately evaluate the product integral as shown in Fig. 4. In addition, for circular Gaussians, the distance in the angular domain (i.e. $\theta_{i}-\theta_{l}$ and $\left.\phi_{i}-\phi_{k}\right)$ matters instead of the spherical signed distance.

Instead of SSDF, our method employs signed distance functions $d\left(\theta_{i}, \phi_{i}\right)$ to compute the product integral of two different (i.e. anisotropic) circular Gaussians and the visibility function. Our method precomputes the visibility function $V\left(\theta_{i}, \phi_{i}\right)$ at each vertex, then the signed distance function $d\left(\theta_{i}, \phi_{i}\right)$ is calculated from $V$ as follows:

$d\left(\theta_{i}, \phi_{i}\right)=\left\{\begin{array}{l}+\min _{V(\theta, \phi)=0} \sqrt{\left(\theta_{i}-\theta\right)^{2}+\left(\phi_{i}-\phi\right)^{2}},\left(V\left(\theta_{i}, \phi_{i}\right)=1\right) \\ -\min _{V(\theta, \phi)=1} \sqrt{\left(\theta_{i}-\theta\right)^{2}+\left(\phi_{i}-\phi\right)^{2}},\left(V\left(\theta_{i}, \phi_{i}\right)=0\right) .\end{array}\right.$

Since $d\left(\theta_{i}, \phi_{i}\right)$ is a signed distance function, its gradient $\nabla d\left(\theta_{i}, \phi_{i}\right)$ points to the nearest point on the visibility boundary where the visibility function $V$ changes, and the gradient $\nabla d\left(\theta_{i}, \phi_{i}\right)$ is equal to the normal at the nearest point on the visibility boundary (see Fig. 4). By exploiting this, our method approximates the visibility function near the peak $\left(\theta_{l}, \phi_{k}\right)$ of two circular Gaussians with a linear visibility function $V^{\prime}\left(\theta_{i}, \phi_{i}\right)$ as:

$V^{\prime}\left(\theta_{i}, \phi_{i}\right)=\left\{\begin{array}{c}1, \frac{\partial d}{\partial \theta_{i}}\left(\theta_{i}-\theta_{l}\right)+\frac{\partial d}{\partial \phi_{i}}\left(\phi_{i}-\phi_{k}\right)+d\left(\theta_{l}, \phi_{k}\right)>0 \\ 0, \frac{\partial d}{\partial \theta_{i}}\left(\theta_{i}-\theta_{l}\right)+\frac{\partial d}{\partial \phi_{i}}\left(\phi_{i}-\phi_{k}\right)+d\left(\theta_{l}, \phi_{k}\right)<0 .\end{array}\right.$

The triple product integral $I$ of two circular Gaussians and the linear visibility function is then calculated by:

$$
I=\iint_{-\frac{\pi}{2}}^{\frac{\pi}{2}} g^{c}\left(\theta_{i} ; \theta_{l}, \sigma_{l}\right) g^{c}\left(\phi_{i} ; \phi_{k}, \sigma_{k}^{\prime}\right) V^{\prime}\left(\theta_{i}, \phi_{i}\right) d \theta_{i} d \phi_{i} .
$$

Although the integral $I$ can be precomputed and stored in a look-up table as $I\left(\theta_{l}, \phi_{k}, \sigma_{l}, \sigma_{k}^{\prime}, d, \nabla d\right)$, it would be a highdimensional table and therefore the precomutation time and storage cost would be prohibitive.

To reduce the dimensionality of the look-up table, our method eliminates $\theta_{l}$ and $\phi_{k}$ from $I$ by approximating the integral $I$ with the integral of two circular Gaussians without occlusion, multiplied by the ratio of occluded two circular

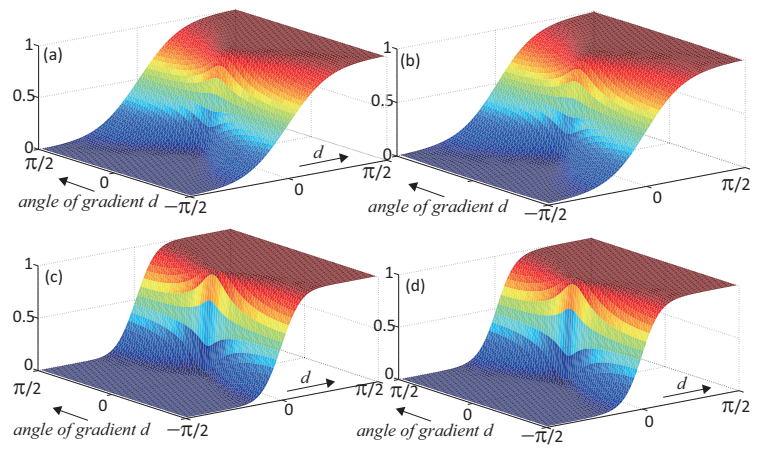

Figure 5: Visualization of $H\left(\sigma_{l}, \sigma_{l}^{\prime}, d, \nabla d\right)$. Top: $\sigma_{l}=$ $1 / 4 \sqrt{2}, \sigma_{l}^{\prime}=1 / \sqrt{2}$, bottom: $\sigma_{l}=1 / 16, \sigma_{l}^{\prime}=1 / 2 \sqrt{2}$. Left: $H\left(\sigma_{l}, \sigma_{l}^{\prime}, d, \nabla d\right)$, right: our sigmoid approximation. The vertical axis (and the color in the graph) represents the value of $H$ in $(a)(c)$ or the value of our sigmoid approximation in $(b)(d)$. The maximum error between $(a)$ and $(b)$ is 0.0093 , that between $(c)$ and $(d)$ is 0.0094 .

Gaussians with zero means $g^{c}\left(\theta_{i} ; 0, \sigma_{l}\right) g^{c}\left(\phi_{i} ; 0, \sigma_{k}^{\prime}\right)$ as:

$$
\begin{aligned}
I & \approx \iint_{-\frac{\pi}{2}}^{\frac{\pi}{2}} g^{c}\left(\theta_{i} ; \theta_{l}, \sigma_{l}\right) g^{c}\left(\phi_{i} ; \phi_{k}, \sigma_{k}^{\prime}\right) d \theta_{i} d \phi_{i} \\
& \times \frac{\iint_{-\frac{\pi}{2}}^{\frac{\pi}{2}} g^{c}\left(\theta_{i} ; 0, \sigma_{l}\right) g^{c}\left(\phi_{i} ; 0, \sigma_{k}^{\prime}\right) V^{\prime}\left(\theta_{i}+\theta_{l}, \phi_{i}+\phi_{k}\right) d \theta_{i} d \phi_{i}}{\iint_{-\frac{\pi}{2}}^{\frac{\pi}{2}} g^{c}\left(\theta_{i} ; 0, \sigma_{l}\right) g^{c}\left(\phi_{i} ; 0, \sigma_{k}^{\prime}\right) d \theta_{i} d \phi_{i}} .
\end{aligned}
$$

In the former double integral in Eq. (12), by approximating the circular Gaussians with Gaussians, the double integral can be calculated on the fly as the following equation:

$$
\begin{aligned}
& \iint_{-\frac{\pi}{2}}^{\frac{\pi}{2}} g^{c}\left(\theta_{i} ; \theta_{l}, \sigma_{l}\right) g^{c}\left(\phi_{i} ; \phi_{k}, \sigma_{k}^{\prime}\right) d \theta_{i} d \phi_{i} \\
\approx & \int_{-\frac{\pi}{2}}^{\frac{\pi}{2}} g\left(\theta_{i} ; \theta_{l}, \sigma_{l}\right) d \theta_{i} \int_{-\frac{\pi}{2}}^{\frac{\pi}{2}} g\left(\phi_{i} ; \phi_{k}, \sigma_{l}^{\prime}\right) d \phi_{i} \\
= & \frac{\pi \sigma_{l} \sigma_{l}^{\prime}}{4}\left[\operatorname{erf}\left(\frac{\pi / 2-\theta_{l}}{\sigma_{l}}\right)-\operatorname{erf}\left(\frac{-\pi / 2-\theta_{l}}{\sigma_{l}}\right)\right] \\
\times & {\left[\operatorname{erf}\left(\frac{\pi / 2-\phi_{k}}{\sigma_{l}^{\prime}}\right)-\operatorname{erf}\left(\frac{-\pi / 2-\phi_{k}}{\sigma_{l}^{\prime}}\right)\right], }
\end{aligned}
$$

where $\operatorname{erf}(x)$ is the error function $\operatorname{erf}(x)=\frac{2}{\sqrt{\pi}} \int_{0}^{x} e^{-t^{2}} d t$, and $\sigma_{l}^{\prime}=\sigma_{k} / \sqrt{\cos \theta_{l} \cos \theta_{k}}$, respectively.

In the latter component of $I$ in Eq. (12), our method can now precompute the 4D function $H\left(\sigma_{l}, \sigma_{l}^{\prime}, d, \nabla d\right)$. We have observed that the 1D function $h(d)=H\left(\sigma_{l}, \sigma_{l}^{\prime}, d, \nabla d\right)$ for fixed $\sigma_{l}, \sigma_{l}^{\prime}, \nabla d$ can be accurately approximated with a sigmoid function. That is,

$$
H\left(\sigma_{l}, \sigma_{l}^{\prime}, d, \nabla d\right) \approx \frac{1}{1+e^{-\kappa\left(\sigma_{l}, \sigma_{l}^{\prime}, \nabla d\right) d}},
$$

where $\kappa\left(\sigma_{l}, \sigma_{l}^{\prime}, \nabla d\right)$ is the gain of the sigmoid function. Our method fits $H$ with the sigmoid function in Eq. (14) by us- 


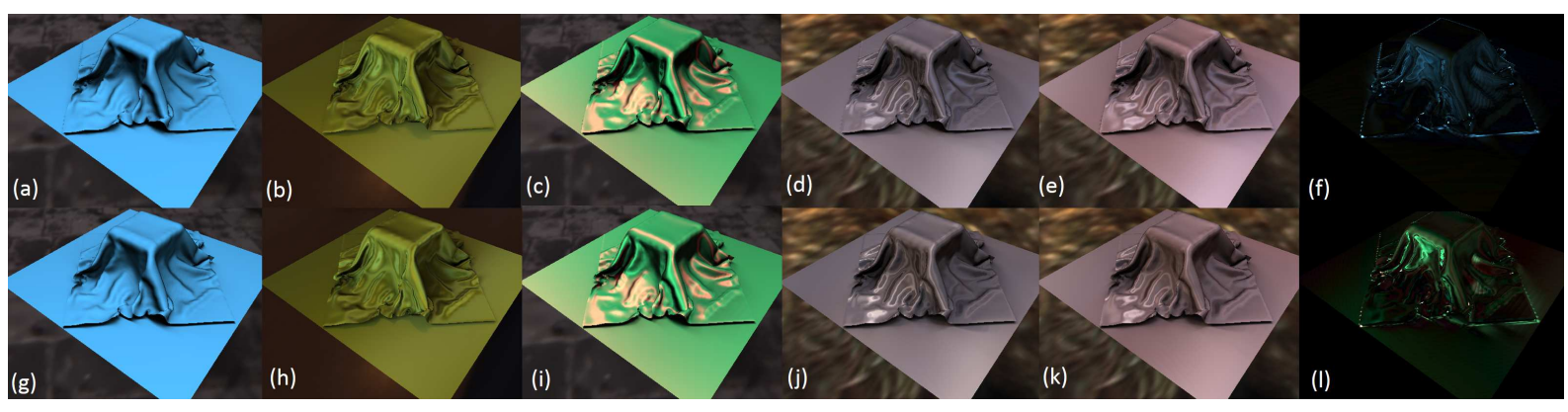

Figure 6: Comparisons of images rendered by using our method (top row) and reference images (bottom row) for various cloth fabrics $((a)(g)$ :linen plain, $(b)(h)$ : silkcrepe de chine, $(c)(i)$ :silk shot fabric, $(d)(j)$ : front face of polyester satin charmeuse, $(e)(k)$ : back face of polyester satin charmeuse) and various environment lightings. The reference images are rendered with direct illumination by using ray tracing (tracing $6 \times 32 \times 32$ rays per pixel to sample incident radiance). The parameters of scattering functions and the tangent curves we used are shown in [Sad11]. $(f)$ and $(l)$ are difference images (scaled by 4) between (a) and $(g)$, and between $(c)$ and $(i)$, respectively.

ing the least square method and stores $\kappa\left(\sigma_{l}, \sigma_{l}^{\prime}, \nabla d\right)$ in the 3D texture. Fig. 5 shows examples of $H$ and its sigmoid approximation. As shown in Fig. 5, our sigmoid approximation matches to the ground truth well.

\section{Results and Discussions}

We have implemented our rendering algorithm on a PC with an Intel Core i7-2700K CPU (3.5GHz) and an NVIDIA GeForce GTX Titan GPU. The resolutions of images in Figs. 1 and 7 are $640 \times 480$, and that for Fig. 6 is $512 \times 384$. Our method calculates the outgoing radiance $L\left(\boldsymbol{\omega}_{o}\right)$ per pixel by using GLSL(OpenGL Shading Language). The rendering performance is reported in Table 1 . The environment lighting is approximated by $10 \mathrm{SGs}$. The rendering performance mainly depends on the number of sampled tangent vectors $\left|C_{1}\right|$ and $\left|C_{2}\right|$. For each vertex of cloth fabric models, SDF $d$ is sampled at $512 \times 512$ directions on the hemisphere and down-sampled to $128 \times 128$ directions. The precomputed SDF is compressed by using PCA with 48 PCA terms, and SDF is interpolated at each pixel at run-time. The compressed SDF for Fig. 1 is $9.26 \mathrm{MB}$ and that for Fig. 7 is $6.02 \mathrm{MB}$. The gain of sigmoid function $\mathrm{\kappa}\left(\sigma_{l}, \sigma_{l}^{\prime}, \nabla d\right)$ is sampled at $32 \times 32 \times 64$.

Fig. 6 shows the comparisons between our method (top row) and the reference images (bottom row) rendered by using ray tracing for various cloth fabrics and environment lighting. The computational times for ray tracing range from $23 \mathrm{~min}$ to $71 \mathrm{~min}$, while those of our method are 11 to 16 fps (frames per second). As shown in Fig. 6, our method can render plausible images of cloth fabrics comparable to the reference images at interactive frame rates.

Fig. 7 shows an example of interactive editing of scattering parameters of cloth fabrics. By editing $A, \gamma_{s}$, and $\gamma_{v}$ parameters that control surface scattering function $f_{r, s}$ and volume scattering function $f_{r, v}$ for two threads, the visual appearance, especially the specular highlight, changes.
Table 2 reports the precomputed data sizes of $T_{\mathbf{t}, \sigma}$ for various cloth fabrics. The numbers of truncated singular values for $T_{\mathbf{t}, \sigma}$ are determined so that the cumulative contribution ratios exceed $90 \%$. To compress $T_{\mathbf{t}, \sigma}$ by using SVD, $\boldsymbol{\xi}_{k}$ and $\boldsymbol{\omega}_{o}$ are sampled for $48 \times 48$ directions. $\mathbf{t}$ is sampled for $\left|C_{1}\right|$ and $\left|C_{2}\right|$ tangent vectors (listed in Table. 1) and $\sigma$ is sampled from $1 / \sqrt{2}$ to $1 / 16 \sqrt{2}$ logarithmically. The precomputation time for $T_{\mathbf{t}, \sigma}$ ranges from $16 \mathrm{~min}$ to $43 \mathrm{~min}$, and that for SVD compression ranges from about 2 hours to 5 hours, which mainly depend on the number of sampled tangent vectors.

To validate our linear visibility approximation with gradient to integrate anisotropic functions, we compare our method with the rendering method for anisotropic spherical Gaussians (ASG) [XSD*13] in Fig. 8. The incident lighting is represented by a single ASG $G\left(\boldsymbol{\omega}_{i} ;[\mathbf{x}, \mathbf{y}, \mathbf{z}],[\lambda, \mu]\right)=$ $\max \left(\boldsymbol{\omega}_{i} \cdot \mathbf{z}, 0\right) \cdot e^{-\lambda\left(\boldsymbol{\omega}_{i} \cdot \mathbf{x}\right)^{2}-\mu\left(\boldsymbol{\omega}_{i} \cdot \mathbf{y}\right)^{2}}$, where $\mathbf{x}, \mathbf{y}, \mathbf{z}$ are orthonormal vectors, $\lambda$ and $\mu$ are bandwidth parameters. In the ASG rendering, to integrate the product of ASG and the visibility function, ASG $G\left(\boldsymbol{\omega}_{i} ;[\mathbf{x}, \mathbf{y}, \mathbf{z}],[\lambda, \mu]\right)$ is approximated by an isotropic spherical Gaussian with the effective bandwidth $\sqrt{\lambda \mu}$. Then the product integral of approximated isotropic spherical Gaussian and the visibility function is computed by using the previous method [WRG*09]. In Fig. 8, $\lambda$ and $\mu$ are set to 10 and 1000 in the top row and vice versa in the bottom row. These ASG lights represent distant lighting through a narrow window or from distant linear light sources. The leftmost column in Fig. 8 shows the reference images rendered by using ray tracing, the center column rendered with our method, and the rightmost column rendered with ASG. Since the ASG rendering method uses the effective bandwidth, Fig. 8(c) is identical to (f), and anisotropic blurred soft shadows cannot be represented. On the other hand, our method can represent anisotropic blurred soft shadows especially from the spout and the handle of the teapot. The blurred soft shadows rendered by using our method, however, slightly spread for thin objects such as the spout and the handle, since the gradient for thin objects changes dras- 


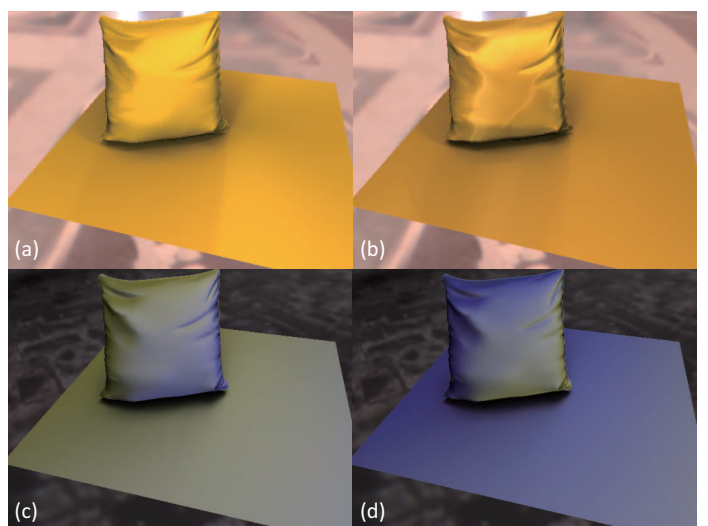

Figure 7: Editing parameters of scattering functions for $(a)(b)$ silk crepe de chine and $(c)(d)$ linen plain. $(a)(b)$ : by editing $\gamma_{s}$, and $\gamma_{r}$ for two threads, the visual appearance of pillow model changes from matte to glossy. $(c)(d)$ : changing albedo A of two threads (yellow and blue) each other.

tically. This can be alleviated by smoothing the SDF, but it remains as future work.

We analyze the approximation error in Eq. (12) for simple linear visibility function that returns 1 if $\theta>0$ and returns 0 otherwise. We calculated root mean square errors (RMSEs) between $I$ and approximation of $I$ in Eq. (12) for various mean parameters $\left(\theta_{l}\right.$ and $\left.\phi_{k}\right)$, which are uniformly sampled from $-\pi / 2$ to $\pi / 2$. For several bandwidth parameters of circular Gaussians, RMSEs are $1.5514 e^{-4}, 4.0907 e^{-5}$, $0.0049,1.7207 e^{-8}$, and 0.0035 when bandwidth parameters $\left(\sigma_{l}, \sigma_{k}^{\prime}\right)$ are set to $(1 / 2,1 / 2),(1 / 2,1 / 8),(1 / 2,1 / 32)$, $(1 / 8,1 / 2)$, and $(1 / 32,1 / 2)$, respectively. As shown in this analysis, our approximation method in Eq. (12) works well for various circular Gaussians.

Although our method can render cloth fabrics under environment lighting interactively, our method has several limitations. Firstly, our method is limited to static scenes in order to precompute SDF. Secondly, our current implementation does not handle interreflections of cloth fabrics. We have investigated the effects of indirect illumination by using path tracing up to 5 bounces in Fig. 9. Although the bottom of the linen pillow looks slightly brighter due to indirect illumination, the effect of indirect illumination seems small in our experiments. However, in certain cases (e.g. indoor scene including multiple cloth fabrics), the effects of indirect illumination may not be small. It would be possible to calculate interreflected light between cloth fabrics with fixed parameters of scattering functions, computing interreflected light of cloth fabrics with dynamic BRDFs remains a challenging problem.

\section{Conclusion and Future Work}

We have proposed an interactive rendering method of cloth fabrics for microcylinder appearance model under environ-

(C) 2014 The Author(s)

Computer Graphics Forum (c) 2014 The Eurographics Association and John Wiley \& Sons Ltd.

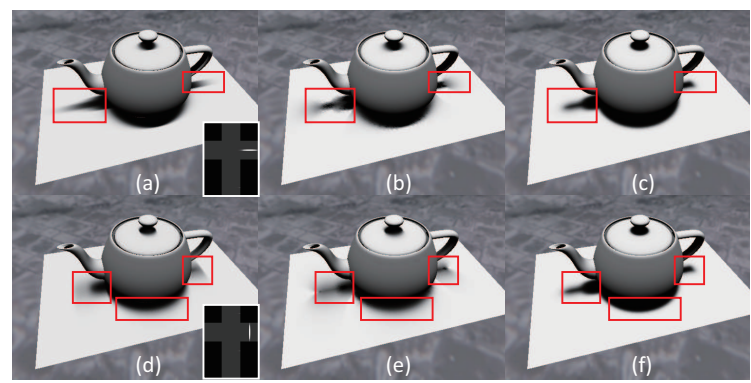

Figure 8: Comparison between ASG and our method. The incident light is represented by a single ASG with $(\lambda, \mu)=$ $(10,1000)$ in the top row and $(\lambda, \mu)=(1000,10)$ in the bottom row, (shown in insets of $(a)$ and $(d)$ ). Due to the anisotropic lighting, the soft shadows are blurred anisotropically as shown in reference images $(a)$ and $(d)$ (especially in the red frames). (b)(e) our method can represent anisotropic blurred shadows from the spout and the handle compared to (c) and (f) with isotropic spherical Gaussians with effective bandwidth $\sqrt{\lambda \mu}$.

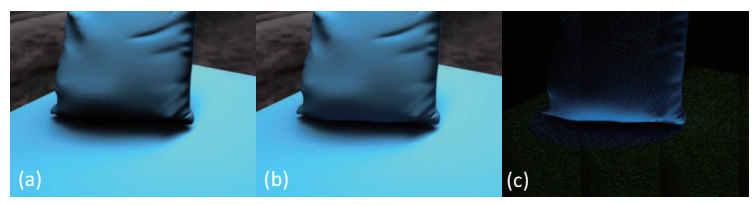

Figure 9: Effects of indirect illumination. (a) direct illumination only $(b)$ with indirect illumination (c) difference image scaled by 4 .

ment lighting. The quadruple product integral of SG, weighting function, scattering function, and visibility function is approximated by the product of SG convolved weighting function and the triple product integral. The SG convolved weighting function can be precomputed for various cloth fabrics and evaluated easily at run-time. The triple product integral of two circular Gaussians and the visibility function is efficiently calculated by using SDF and its gradient. Our method enables the users to edit the parameters of scattering functions that control the visual appearances of cloth fabrics interactively under all-frequency environment lighting.

In future work, we would like to take into account indirect illumination. Moreover, we would like to extend our method to handle dynamic scenes of cloth fabrics.

Table 1: Rendering performance. $\left|C_{1}\right|$ and $\left|C_{2}\right|$ are numbers of sampled tangent vectors of thread 1 and 2 , respectively.

\begin{tabular}{|c|c|c|c|}
\hline Figure No. & Cloth & $\left|C_{1}\right|+\left|C_{2}\right|$ & fps \\
\hline Fig. 1(a)/6(a)/7 & linen & 6 & $13 / 16 / 11$ \\
\hline Fig. 1(b)/6(d)(e) & polyester & 16 & $9 / 11$ \\
\hline Fig. 1(c)/6(b)/7 & silk & 10 & $10 / 16 / 12$ \\
\hline Fig. 1(d) & velvet & 8 & 11 \\
\hline Fig. 6(c) & silkshot & 10 & 13 \\
\hline
\end{tabular}


Table 2: Data sizes of precomputed table T. The minimum/maximum/average numbers of singular values for various $\mathbf{t}$ and $\sigma$ are listed.

\begin{tabular}{|c|c|c|}
\hline Cloth & Data size & $\begin{array}{c}\text { \# of singular values } \\
\text { (min/max/ave) }\end{array}$ \\
\hline linen & $29.6 \mathrm{MB}$ & $1 / 24 / 8.8$ \\
\hline silk & $40.5 \mathrm{MB}$ & $1 / 19 / 7.2$ \\
\hline silk shot & $81.1 \mathrm{MB}$ & $1 / 44 / 14.4$ \\
\hline polyester (front) & $66.9 \mathrm{MB}$ & $1 / 21 / 7.4$ \\
\hline polyester (back) & $66.0 \mathrm{MB}$ & $1 / 21 / 7.3$ \\
\hline velvet & $41.7 \mathrm{MB}$ & $1 / 23 / 9.3$ \\
\hline
\end{tabular}

\section{Acknowledgements}

We would like to thank all the reviewers for their useful comments. This research was partially supported by JSPS KAKENHI Grant Number 24700093 and 13324688.

\section{References}

[AMTF03] Adabala N., Magnenat-Thalmann N., Fei G.: Visualization of woven cloth. In Proc. of Eurographics Symposium on Rendering (2003), pp. 178-185. 2

[APS00] AShikMin M., PREMOŽE S., Shirley P.: A microfacet-based brdf generator. In Proc. of SIGGRAPH'OO (2000), pp. 65-74. 2

[DLHpS01] Daubert K., Lensch H. P. A., Heidrich W., PETER SEIDEL H.: Efficient cloth modeling and rendering. In $E u$ rographics Workshop on Rendering (2001), pp. 63-70. 2

[HR13] HAŠAN M., RAMAMOORTHI R.: Interactive albedo editing in path-traced volumetric materials. ACM Transactions on Graphics 32, 2 (2013), 11:1-11:11. 2

[IDN12] IWASAKI K., Dobashi Y., Nishita T.: Interactive biscale editing of highly glossy materials. ACM Transactions on Graphics 31, 6 (2012), 144:1-144:7. 5

[IFDN12] IWASAKI K., FURUYA W., Dobashi Y., Nishita T.: Real-time rendering of dynamic scenes under all-frequency lighting using integral spherical gaussian. Computer Graphics Forum 31, 2 (2012). 5

[IM12] IRAWAN P., Marschner S.: Specular reflection from woven cloth. ACM Transactions on Graphics 31, 1 (2012), 11:111:20. 2

[JAM*10] Jakob W., Arbree A., Moon J. T., Bala K., MARSCHNER S.: A radiative transfer framework for rendering materials with anisotropic structure. ACM Transactions on Graphics 29, 4 (2010), 53:1-53:13. 2

[Kan10] KANG Y.-M.: Realtime rendering of realistic fabric with alternation of deformed anisotropy. In Proc. of Motion in Games (2010), pp. 301-312. 2

[MMK04] Muller G., Meseth J., Klein R.: Fast environmental lighting for local-pca encoded btfs. In Proc. of Computer Graphics International (2004), pp. 198-205. 2

[RZL*10] ReN Z., Zhou K., Li T., HuA W., Guo B.: Interactive hair rendering under environment lighting. ACM Transactions on Graphics 29, 4 (2010), 55:1-55:8. 2

[Sad11] SADEghi I.: Controlling the Appearance of Specular Microstructures. PhD thesis, UC San Diego, 2011. 1, 3, 6
[SBdDJ13] SAdeghi I., Bisker O., DE DeKen J., Jensen H. W.: A practical microcylinder appearance model for cloth rendering. ACM Transactions on Graphics 32, 2 (2013), 14:114:12. 1, 2, 3

[SKS02] Sloan P.-P., Kautz J., Snyder J.: Precomputed radiance transfer for real-time rendering in dynamic, lowfrequency lighting environments. ACM Trans. Graph. 21, 3 (2002), 527-536. 2

[SKZ11] SchröDER K., KleIn R., ZINKE A.: A volumetric approach to predictive rendering of fabrics. Computer Graphics Forum 30, 4 (2011), 1277-1286. 2

[SSK03] Sattler M., Sarlette R., Klein R.: Efficient and realistic visualization of cloth. In Eurographics Symposium on Rendering (2003), pp. 167-177. 2

[Wat92] Westin S. H., Arvo J. R., Torrance K. E.: Predicting reflectance functions from complex surfaces. In Proc. of SIGGRAPH'92 (1992), pp. 255-264. 2

[WPC*13] Wang R., Pan M., Chen W., Ren Z., Zhou K., HUA W., BAO H.: Analytic double product integrals for allfrequency relighting. IEEE Transactions on Visualization and Computer Graphics 19, 7 (2013), 1133-1142. 5

[WRG*09] Wang J., Ren P., Gong M., SNyder J., Guo B.: All-frequency rendering of dynamic, spatially-varying reflectance. ACM Transactions on Graphics 28, 5 (2009), 133:1133:10. 5, 6

[WZT*08] WANG J., Zhao S., TONG X., SNyder J., Guo B.: Modeling anisotropic surface reflectance with example-based microfacet synthesis. ACM Trans. Graph. 27, 3 (2008), 41:1-41:9.

[XCL*01] Xu Y.-Q., Chen Y., Lin S., Zhong H., Wu E., GUO B., SHum H.-Y.: Photorealistic rendering of knitwear using the lumislice. In Proc. of SIGGRAPH2001 (2001), pp. 391398. 2

[XJF*08] Xu K., Jia Y.-T., Fu H., Hu S., TaI C.-L.: Spherical piecewise constant basis functions for all-frequency precomputed radiance transfer. IEEE Transactions on Visualization and Computer Graphics 14, 2 (2008), 454-467. 5

[XMR*11] XU K., Ma L.-Q., Ren B., Wang R., HU S.-M.: Interactive hair rendering and appearance editing under environment lighting. ACM Transactions on Graphics 30, 6 (2011), 173:1-173:10. 2, 4

[XSD*13] Xu K., Sun W.-L., Dong Z., Zhao D.-Y., Wu R.D., HU S.-M.: Anisotropic spherical gaussians. ACM Transactions on Graphics 32, 6 (2013), 209:1-209:11. 6

[YYTI92] YASUda T., YoKoI S., TORIWAKI J.-I., INAGAKI $\mathrm{K}$.: A shading model for cloth objects. IEEE Computer Graphics and Applications 12, 6 (1992), 15-24. 2

[ZHRB13] ZhaO S., HaŠAN M., RAMAMOORTHI R., BALA K.: Modular flux transfer: Efficient rendering of high-resolution volumes with repeated structures. ACM Transactions on Graphics 32, 4 (2013), 131:1-131:12. 2

[ZJMB11] ZhaO S., JaKob W., Marschner S., Bala K.: Building volumetric appearance models of fabric using micro ct imaging. ACM Transactions on Graphics 30, 4 (2011), 44:144:10. 2

[ZJMB12] Zhao S., Jakob W., Marschner S., Bala K. Structure-aware synthesis for predictive woven fabric appearance. ACM Transactions on Graphics 31, 4 (2012), 75:1-75:10. 2 